\title{
Contrôle de l'envahissement des pâturages par la brousse
}

\author{
par Z. DERBAL
}

Les pâturages du Centre Fédéral de Recherches Zootechniques à Bamako (Soudan français) représentent deux types que l'on rencontre au Soudan: - la savane arbustive ou arborescente, qui correspond aux zones hautes, jamais inondées;

- la savane herbeuse, qui correspond aux terres basses des bords du fleuve, partiellement inondées à l'époque de la crue.

La Station se trouve dans la zone soudanienne, caractérisée par un climat du type tropical, avec des pluies assez abondantes (1.000- $1.400 \mathrm{~mm}$ ), qui tombent régulièrement pendant l'hivernage, de juin à octobre. Le reste de l'année correspond à la saison sèche.

Les températures maxima sont enregistrées en avril-mai $\left(44^{\circ} \mathrm{C}\right)$ et les minima en décembre-janvier $\left(12-14^{\circ} \mathrm{C}\right.$, exceptionnellement $\left.7^{\circ} \mathrm{C}\right)$.

Le degré hygrométrique varie de $20 \%$ en saison sèche à $90 \%$ à la fin de la saison des pluies.

Du fait de la brièveté de la saison des pluies, la durée du cycle végétatif des espèces annuelles est réduite. Les plantes se dessèchent rapidement sur pied dès le début de la saison sèche.

Les pâturages des bords du fleuve et des zones basses gardent une certaine fraicheur et restent verts plus longtemps que ceux des zones sèches.

La majeure partie des parcours du Centre Fédéral de Recherches Zootechniques est utilisée comme pâturages naturels. Des essais de création de pâturages artificiels sont en cours depuis 1953.

\section{Pâturages naturels}

Un système de pistes et de larges pare-feu découpe les pâturages du Centre Fédéral en parcelles dont la surface varie de 20 à 40 ha et les protège assez bien contre les feux de brousse.

Une parcelle témoin a été conservée en défense absolue depuis l'installation de la Station en 1952 et, depuis cette date, aucun animal n'y a pénétré; elle n'a été ni brûlée ni traitée.

Bien que certains pâturages et, en particulier, ceux de la zone « savane arbustive », aient été soumis à une certaine charge à l'hectare, ce terrain a été progressivement envahi par des espèces herbacées, buissonnantes et arbustives, assez peu facilement utilisables comme fourrage du fait de leur structure ligneuse. Les principales sont les suivantes:

Combretacées : Combretum spp.

Pteleopsis suberosa,

Guiera senegalensis,

Terminalia,

Anogeissus leiocarpus.

Euphorbiacées : Phyllanthus spp.

Cochlospermacées: Cochlospermum tinctorium.

Convolvulacées : Ipomea repens.

Malvacées : Sida carpinifolia.

Papillionacées : Crotalaria spp., Indigofera spp.

\section{Méthodes d'entretien.}

Pour améliorer les qualités naturelles dos pâturages, on peut avoir recours à des techniques chimiques ou à des traitements et façons culturales pouvant être faits soit à la machine, soit à la main.

a) Procédés chimiques.

N'ont pas été expérimentés.

b) Procédés mécaniques.

Deux machines ont été essayées pour la destruction des arbustes et plantes envahissantes:

- La débroussailleuse-faucheuse, avec scie circulaire horizontale : coupe assez bien les arbustes dont le diametre est inférieur à $6 \mathrm{~cm}$ et le couvert herbeux. Cette machine est un peu lourde et assez difficile à manceuvrer. Elle a l'inconvénient de laisser en place le systeme radiculaire: Les arbustes repoussent rapidement.

- La débroussaillouse "La Landaise »: est formée par un bâti que l'on peut charger et auquel est fixé un axe en acier supportant 6 couteaux parallèles aux génératrices de l'axe.

En avançant, la mactine sectionne les herbes et broussailles et les couteaux pénètrent plus ou moins 
profondément dans le sol suivant que le bâti est plus ou moins chargé.

Les racines sont coupées à une profondeur n'excédant pas $10 \mathrm{~cm}$. meilleure façon d'améliorer les pâturages est de supprimer les arbustes et arbres inutilisables en les sectionnant au-dessous du collet et en éliminant la plus grande partie des grosses racines, en laissant

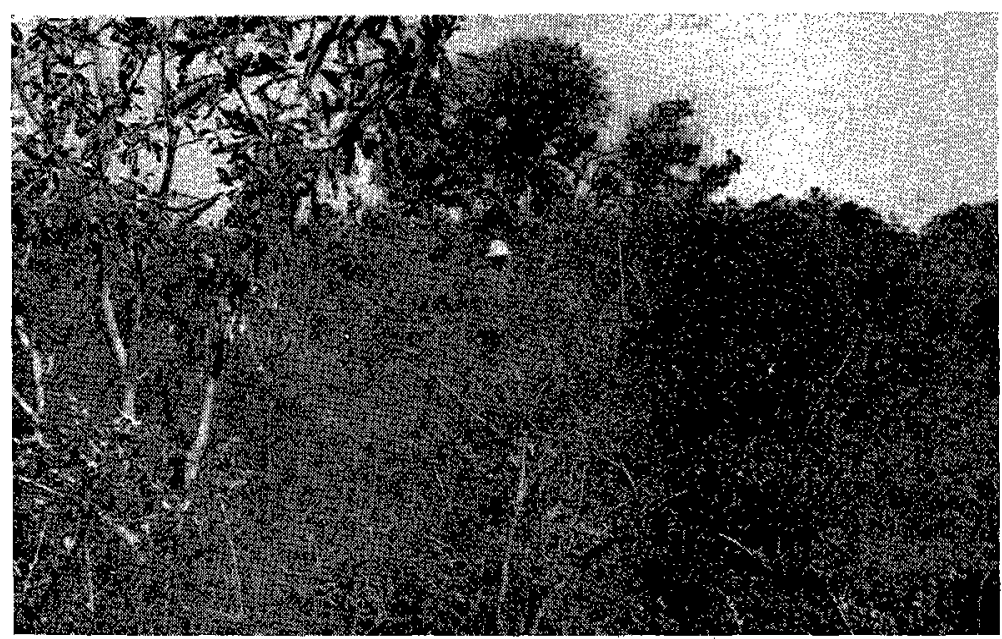

Aspect de la végétation avant débroussaillement.

Sotuba.

Après le passage de la machine, le terrain reste bosselé et légèrement ondulé.

Cette machine, comme la précédente, ne permet pas une amélioration très sensible des qualités du pâturage. de grands arbres et des arbustes de façon à laisser subsister des îlots d'ombre. On transforme la brousse en une espèce de savane-parc.

Les espèces annuelles, ayant à leur disposition un espace vital plus grand, envahissent le pâturage.

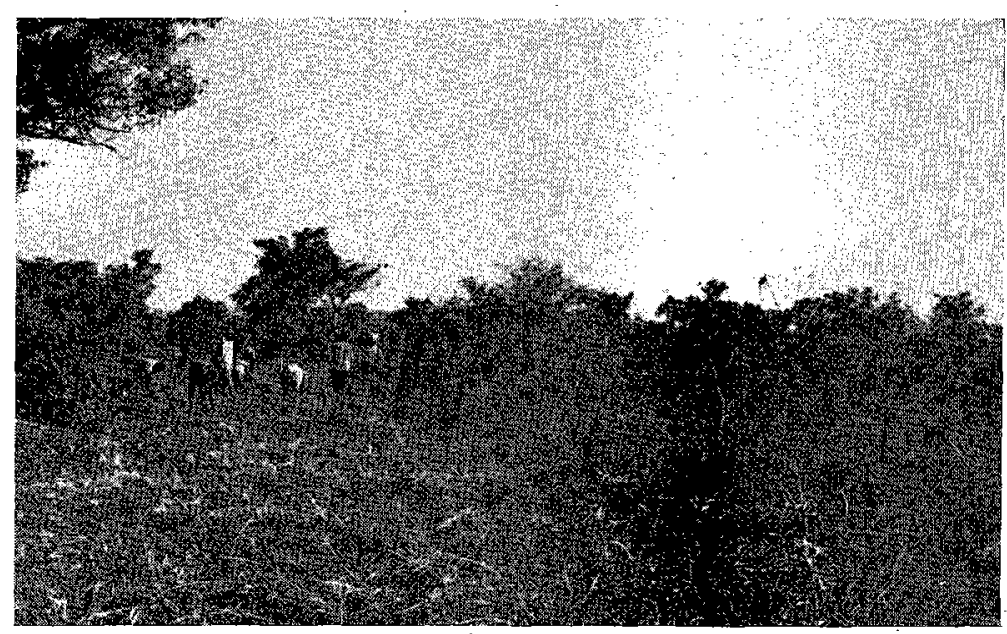

Premiers résultats du débroussaillement par bandes. Sotuba.

c) Procédés manuels:

Bien que l'entretien manuel des pâturages soit assez onéreux, il reste le procédé de choix. La
Chaque année, on élimine à la main les espèces les moins favorables (crotalaires, Guiera senegalensis, Sida carpinifolia). 
d) Procédés mixtes.

Après que l'on a préparé à la main les terrains, comme nous l'avons indiqué ci-dessus, on peut utiliser des machines pour améliorer la qualité du pâturage.

La faucheuse élimine les refus en fin de saison des pluies. On obtient une repousse qui peut être utilisée en début de saison sèche par les animaux.

On peut également entretenir la surface en passant un pulvériseur à disquies, un cultivateur, une herse, qui peuvent être trainés soit par un tracteur, soit par des bceufs.

Nous n'avons pas voulu généraliser cette technique de débroussaillement à de grandes surfaces car nous avons pensé que cela favorisait l'erosion éolienne et modifiait le micro-climat local.

\section{Technique pratique adoptée.}

Chaque parcelle de pâturage est divisée en bandes alternativement débroussées et laissées en nature. Les bandes débroussées ont une largeur de $60 \mathrm{~m}$, les bandes laissées en état une largeur de $30 \mathrm{~m}$.

Avantages de la technique :

L'entretien des parties débrousssées se fait à peu de frais : il suffit d'arracher, à la fin de chaque saison des pluies, les rejets des plantes inutilisables. Si la paille est fauchée en fin de saison des pluies, les risques de feux de brousse sont diminués du fait de la moindre quantité de paille sèche qui reste sur place.

Les refus et les chaumes fournissent de la paille utilisable pour les litières.

Le fauchage en début de saison sèche provoque la repousse assez rapide de certaines espèces vivaces qui sont, en particulier l'Andropogon gayanus var. bisquamulatus, très appréciées par les animaux.

Dans un terrain, l'Andropogon gayanus, fauché à 6 reprises au cours des 8 mois de saison sèche. a donné 6 repousses d'une hauteur moyenne de
$35 \mathrm{~cm}$, ce qui représente, pour 8 mois, un rendement de $950 \mathrm{gr}$ de matière verte pour une touffe d'Andropogon.

\section{Pâturages artificiels}

Des pâturages artificiels ont été créés sur des terrains du Centre Fédéral de Recherches Zootechniques : la première parcelle de 33 ha a été ensemencée en 1954. La parcelle choisie avait été cultivée au cours des années précédentes de la façon suivante :

- Ire année après débroussaillement : une sole de mais et de sorgho avec fumure au fumier de ferme.

- 2* année: une sole de sorgho à grains.

$-3^{*}$ année: jachère avec passage du pulvériseur à disques et du cultivateur.

Le calendrier des travaux a été le suivant:

1. En mai, après la première pluie, l'enfouissement du couvert herbeux a été exécuté avec la déchaumeuse à disques travaillant à $18-20 \mathrm{~cm}$ de profondeur.

$2^{\circ}$ En juin, on a passé une fois le pulvériseur à disques et une fois le cultivateur canadien pour extirper les racines et les mauvaises herbes.

$3^{\circ}$ Début juillet, on a semé à la volée, à raison de $16 \mathrm{~kg}$ à l'hectare, un mélange de graines sèches contenant $6 \mathrm{~kg}$ de graines de graminées spontanées pour $10 \mathrm{~kg}$ de graines de Sudan grass.

Le mélange contenait les espèces suivantes:

Pennisetum polystachyum,

Setaria sphacelata,

Paspalum scrobiculatum,

Eleusine indica,

Echinochloa colona,

Andropogon sudanensis.

$4^{\circ}$ Le semis a été suivi d'un hersage léger pour enterrer les graines.

Prix de revient des façons culturales

\begin{tabular}{|c|c|c|c|c|c|c|c|}
\hline & $\begin{array}{l}\text { NOMBRE } \\
\text { journées } \\
\text { tracteur }\end{array}$ & $\begin{array}{l}\text { NOMBRE } \\
\text { journées } \\
\text { chauffeur }\end{array}$ & $\begin{array}{l}\text { NOMRRE } \\
\text { journées } \\
\text { manœuvres }\end{array}$ & $\begin{array}{c}\text { PRIX } \\
\text { du carburant }\end{array}$ & $\begin{array}{c}\text { SALAIRE } \\
\text { du chauffeur }\end{array}$ & $\begin{array}{c}\text { SALAIRE } \\
\text { des manœuures }\end{array}$ & TOTAUX \\
\hline Pulvériseur .... & 8 & 8 & 0 & 8.960 & 2.056 & & 11.016 \\
\hline Herse & 3 & 3 & 6 & 3.680 & 771 & 834 & 5.285 \\
\hline Semis & 2 & 2 & 4 & 1.600 & 514 & 556 & 2.670 \\
\hline & & & & & & & $\begin{array}{c}18.971 \\
\text { Soit : } 575 \text { fr. } \\
\text { à l'hcctare. }\end{array}$ \\
\hline
\end{tabular}




\section{Observations effectuées}

$I^{\text {re }}$ année : la levée a été très bonne, cependant la densité d'Andropogon sudanensis n'a pas été proportionnelle à la quantité des graines présentes dans le mélange.

Pendant l'hivernage, lés espèces semées restent dominantes.

Au début de la saison sèche, les herbes adventices apparurent, surtout les crotalaires qui, chaque année, envahissent tous les terrains de cultures et les jachères du Centre. Ces plantes ont été arrachées avant fructification.

Un essai de rendement, fait le 2 septembre, a montré que l'on pouvait récolter 20,5 t d'herbe à l'hectare.

Les herbes semées se sont desséchees au début de la saison sèche, dans l'ordre suivant:

Sudan grass,

Echinochloa colona,

Pennisetum polystachyum,

Setaria pallidifusca et sphacelata,
Eleusine indica,

Paspalum scrobiculatum.

$z^{\circ}$ année: la reprise de la végétation, ainsi que le rendement, furent satisfaisants. Cependant, vers la fin de l'hivernage, de mauvaises herbes:

Sida carpinifolia,

Crotalaria retusa,

Acanthospermum hispidum,

Ipomea repens,

apparurent en grand nombre. Leur répartition dépendait de la distribution de l'eau à la surface de la prairie.

Dans les parties non inondées en hivernage, les crotalaires se développent d'abord, suivies de Sida carpinifolia. Dans les parties inondées, Ipomea domine au dóbut, remplacóe en saison sèche par des crotalaires.

(Travail du Centre Fédéral de Recherches Zootechniques de l'A.O.F.).

\section{SUIMMARY}

\section{Control of bush in pastures}

In the Sotuba Livestock Research Station (French Sudan, West Africa), trials have been made to improve natural pastures.

Various mechanical technics have been tried and the resulting changes on the grassland flora are described.

Other results obtained on cultivated pastures and their cost are noted.

\section{RESUIMEN}

\section{Control de la invasión de los pastos por el tamojal}

En el Centro Federal de Investigaciones Zootecnicas del A.O.F. de Sotuba (Sudán Francés) se han realizado ensayos de mejora de los pastos naturales.

Se han experimentado divorsos proccdimientos mocánicos y se describe la evolución consiguicnte de la flora.

Se han hecho observaciones sobre los resultados obtenidos en la creación de pastos artificiales y sobre su precio de coste. 